\title{
The effects of early vitamin A supplementation on the prevention and treatment of bronchopulmonary dysplasia in premature infants: a systematic review and meta-analysis
}

\author{
Li Huang, Diqing Zhu, Gaofeng Pang \\ Department of Pediatrics, The Third Affiliated Hospital of Soochow University, Changzhou, China \\ Contributions: (I) Conception and design: L Huang, G Pang; (II) Administrative support: D Zhu; (III) Provision of study materials or patients: All \\ authors. (IV) Collection and assembly of data: All authors; (V) Data analysis and interpretation: All authors. (VI) Manuscript writing: All authors; (VII) \\ Final approval of manuscript: All authors. \\ Correspondence to: Gaofeng Pang. No. 185 Juqian Street, Tianning District, Changzhou City 213003, China. Email: czyypp008@126.com.
}

Background: Bronchopulmonary dysplasia (BPD) is a common and unique chronic disease of premature
infants with a high mortality rate. A large number of studies have shown that vitamin A supplementation has
a better effect on the prevention and treatment of BPD.

Methods: Randomized controlled trials (RCTs) on early vitamin A supplementation for the prevention and treatment of BPD were searched in PubMed, EMBASE, and the Cochrane Library database. The search time was from the establishment of the database to July 28, 2021. Two investigators performed standard independent screening trials of the literature according to the inclusion and exclusion criteria. The methodology of the included articles was evaluated, and the data were extracted. If the opinions of the 2 investigators were not consistent, a third party could be consulted. The Cochrane systematic review manual was used to analyze the bias of the included studies, and the RevMan 5.3 software was used to perform the meta-analysis.

Results: In the experimental group, the BPD incidence [-0.71, 95\% confidential interval (CI): -0.34 to -0.00 ; $\mathrm{Z}=1.98 ; \mathrm{P}=0.05$ ], 28-day oxygen uptake rate (0.81, 95\% CI: 0.38-1.730; $\mathrm{Z}=0.53 ; \mathrm{P}=0.59)$, 36-week survival rate (1.08, 95\% CI: 0.80-1.46; $\mathrm{Z}=0.49 ; \mathrm{P}=0.62)$, incidence of patent ductus arteriosus (0.77, 95\% CI: 0.27-2.21; $\mathrm{Z}=0.48 ; \mathrm{P}=0.63)$, days of mechanical ventilation $(0.02,95 \% \mathrm{CI}:-1.46-1.49 ; \mathrm{Z}=0.02 ; \mathrm{P}=0.98)$, and 28-day ventilator use $(0.77,95 \% \mathrm{CI}: 0.31-1.92 ; \mathrm{Z}=0.55 ; \mathrm{P}=0.58)$ were lower than those in the control group, especially the incidence of BPD.

Discussion: Early supplementation of vitamin A showed good efficacy and safety in the prevention and treatment of BPD in premature infants, and can also improve the survival rate of infants. Therefore, it is worthy of clinical application.

Keywords: Bronchopulmonary dysplasia (BPD); premature infants; vitamin A; meta-analysis; efficacy and safety

Submitted Sep 27, 2021. Accepted for publication Nov 12, 2021.

doi: $10.21037 / \mathrm{tp}-21-496$

View this article at: https://dx.doi.org/10.21037/tp-21-496

\section{Introduction}

Bronchopulmonary dysplasia (BPD), also known as chronic lung disease $(\mathrm{CLD})$, is a common and unique chronic disease of premature infants. It mainly induces severe respiratory distress syndrome (RDS) caused by mechanical ventilation (1). BPD can cause neurodevelopmental disorders of premature infants, prolonging the hospital stay and increasing the risk of re-hospitalization. BPD in premature infants not only shows a higher mortality rate, but it may have a lifelong impact on children, which imposes a heavy burden on the physical and mental health of children, their families, and society. With the continuous advancement of perinatal technology, the birth rate and survival rate of very low birth weight and ultra-low birth 
weight infants have not only increased, but the incidence of BPD has also increased (2). Research on the prevention and treatment of BPD appears to be necessary and imminent (3). For many years, researchers have conducted numerous studies on the prevention and treatment of premature infants with BPD. Although some progress has been made, there is still a lack of effective methods and measures for the prevention and treatment of premature infants with BPD (4). Vitamin A deficiency is a global public health problem. Vitamin A is mainly passed from mother to fetus through the placenta in the third trimester. Therefore, most premature infants are deficient in vitamin $\mathrm{A}$. A large number of research results show that the younger the gestational age, the lower the weight, and the more serious the vitamin A deficiency. BPD is also more common in young premature infants, which suggests that the occurrence of BPD may be related to the lack of vitamin A. Researchers compared the plasma vitamin levels in early infants with $\mathrm{BPD}$, and vitamin deficiency was found to be associated with BPD (5). As early as 1987, a double-blind randomized trial proved that vitamin A supplementation can prevent and treat the occurrence of BPD in premature infants. There are also a large number of clinical studies and evidence-based medicine results that have proven this theory in recent years. The mechanisms of vitamin A supplementation in the prevention and treatment of BPD may be as follows. Firstly, vitamin A can regulate and promote the proliferation and differentiation of various lung cells, thereby maintaining the integrity of the airway epithelium. Secondly, vitamin A can enhance the immune function of newborns, thereby further enhancing the newborn's ability to resist disease and damage. Thirdly, vitamin A can promote the synthesis of lung surface active substances. Fourthly, vitamin A has a certain antioxidant effect. Additionally, vitamin A can also promote repair after lung injury (6).

Although there have been numerous studies proving that vitamin $\mathrm{A}$ is a potential effective nutrient for preventing $\mathrm{BPD}$, repeated intramuscular injections are required to achieve a therapeutic effect due to its relatively gentle force. Newborns are fragile and are not suited to long-term repeated intramuscular injections. This is also one of the reasons that hinders the application of vitamin $\mathrm{A}$ in the treatment of premature infants with BPD (7). The related theory that vitamin A can prevent and treat BPD in very low birth weight infants was put forward by researchers 20 years ago. In addition, many clinical studies on oral vitamin A prevention and treatment of BPD are still at the stage of patient recruitment. Therefore, many researchers have questioned the ability of vitamin A to prevent and treat BPD based on the above background and the continuous advancement of perinatal technology (8). For example, some researchers believe that vitamin A supplementation is not only ineffective in preventing and treating BPD, but may also increase the risk of sepsis in children (9). Studies have also shown that there is no correlation between vitamin A deficiency, neonatal death, and the occurrence of BPD $(10,11)$. In summary, it can be known that there is no accurate conclusion yet on the influence of early vitamin A supplementation on the occurrence of BPD. Therefore, this paper searched the articles related to early vitamin A supplementation on the occurrence of BPD and made A detailed analysis on the emergence of key outcome indicators such as oxygen uptake rate at 28 days, mechanical ventilation days, and 36-week survival rate. In order to provide reference and basis for clinical treatment of related diseases. We present the following article in accordance with the PRISMA reporting checklist (available at https://dx.doi. org/10.21037/tp-21-496).

\section{Methods}

\section{Article retrieval}

The search databases were PubMed, EMBASE, the Cochrane Library, and the Chinese Biomedical Literature Database. The search time was from the establishment of the database to July 28, 2021, and the articles were also traced. The retrieval method was a combination of subject words and free words. The English search terms were as follows: bronchopulmonary dysplasia, chronic lung disease, vitamin $\mathrm{A}$, aquasol $\mathrm{A}$, retinol, retinoid, retinoic, infant, newborn, neonate, neonatal, premature, low birth weight, randomized controlled trial, trial, and placebo.

\section{Inclusion and exclusion criteria of the articles}

The inclusion criteria were defined as follows: articles with all the basic information included; articles with a sufficient number of cases; articles which were clinical trials; subjects with a non-congenital abnormality, premature infants with short-term survival difficulty, and gestational age less than 37 weeks; articles with intervention measures including vitamin A supplementation (the drug route, dosage, and course of treatment were not limited) based on routine vitamin A supplementation (breast milk, premature infants milk, intravenous hypernutrition, etc.), and a placebo or no extra vitamin A supplementation in the control group. 
The exclusion criteria were as follows: poor quality articles that had serious flaws in the research design and improper randomization methods that led to a lack of randomness; the same articles which published the largest number of samples multiple times; articles with none of the main or secondary indicators required; and articles with unavailable original texts or sufficient original data was not provided.

\section{Data extraction}

Two researchers independently screened and evaluated the retrieved articles based on the inclusion and exclusion criteria, which was cross checked after data extraction using the same data extraction table. If a unified agreement could not be obtained by these two researchers, it would be discussed and resolved by finding a third party. The main extraction content was as follows: basic information of the included research such as publication time, country, or region; research methods and key elements of bias risk evaluation; characteristics of the research subjects, including baseline data, inclusion and exclusion criteria, and sample size; treatment methods, including administration time, route, dose, and course of treatment; and outcome evaluation indicators and diagnostic criteria.

\section{Clinical effect indicators}

Primary outcome measures were 28-day BPD rate 28-day oxygen rate 36-week mortality secondary outcome measures were 28-day ventilator use number of ventilation days and PDA rate. When the accurate mean and standard deviation were not reported in the included literature, it was estimated by the graphs used in the literature. All data were from the included articles.

\section{Quality assessment}

In order to assess the quality of the review literature, quality was evaluated using the Cochrane Manual for Systematic evaluation of interventions. The evaluation content included the following seven items: (I) which randomization method was used; (II) whether allocation concealment was used; (III) the implementation of blinding between patients and researchers; (IV) evaluation of the effect of blinding; (V) whether the results were complete; (VI) whether the research results were credible; and (VII) other biases. Regarding the randomized controlled trials (RCTs) for item seven above, "satisfied" meant that the bias was relatively low and "unsatisfied" meant that the bias was high and the research did not have sufficient detailed reports. Unknown risk assessment includes random sequence generation, allocation concealment, blinding, and tracking/exit. The four dimensions were scored from 1 to 3 points, which was considered as low quality, and 4-7 points were considered high quality.

\section{Statistical analysis}

In this study, the RevMan 5.3.5 software provided by the Cochrane Collaboration was adopted to analyze the article data. Binary variables were evaluated by relative risk (RR) and odds ratio (OR), while weighted mean difference (WMD) was used to evaluate continuous variables and calculate their $95 \%$ confidence interval (CI). As for the heterogeneity of the literature, the $\chi^{2}$ test was adopted to test the homogeneity of the included studies. If $\mathrm{P}>0.1$ and $\mathrm{I}^{2}<50 \%$, the included articles were considered to be homogeneous or consistent, and the fixed effects model (FEM) was adopted. If $\mathrm{P}<0.1$ and $\mathrm{I}^{2}>50 \%$, the included articles were considered to be heterogeneous, and the random effects model (REM) was used for analysis. The meta-analysis test level was set to $\alpha=0.05$, and $\mathrm{P} \leq 0.05$ meant that the difference was statistically significant.

\section{Results}

\section{Results of article retrieval and the basic characteristics of the included articles}

A total of 465 related articles were retrieved in this study, of which 179 were retrieved from the PubMed database, 154 were retrieved from the EMBASE database, 55 were retrieved from the Cochrane Library database, and 77 were retrieved from the China Biomedical Literature Database. A total of 122 articles were excluded due to duplicated published articles. After the title and abstract were read, 319 articles that obviously did not meet the inclusion criteria were excluded, and 15 articles were eliminated after the full text was read. Finally, six (12-17) articles were included, with a total of 1,499 newborns. For details, refer to the literature screening flowchart shown in Figure 1. The basic information of the included literature is shown in Table 1.

\section{Risk bias assessment of the included articles}

The Cochrane Handbook version 5.3 systematic review 


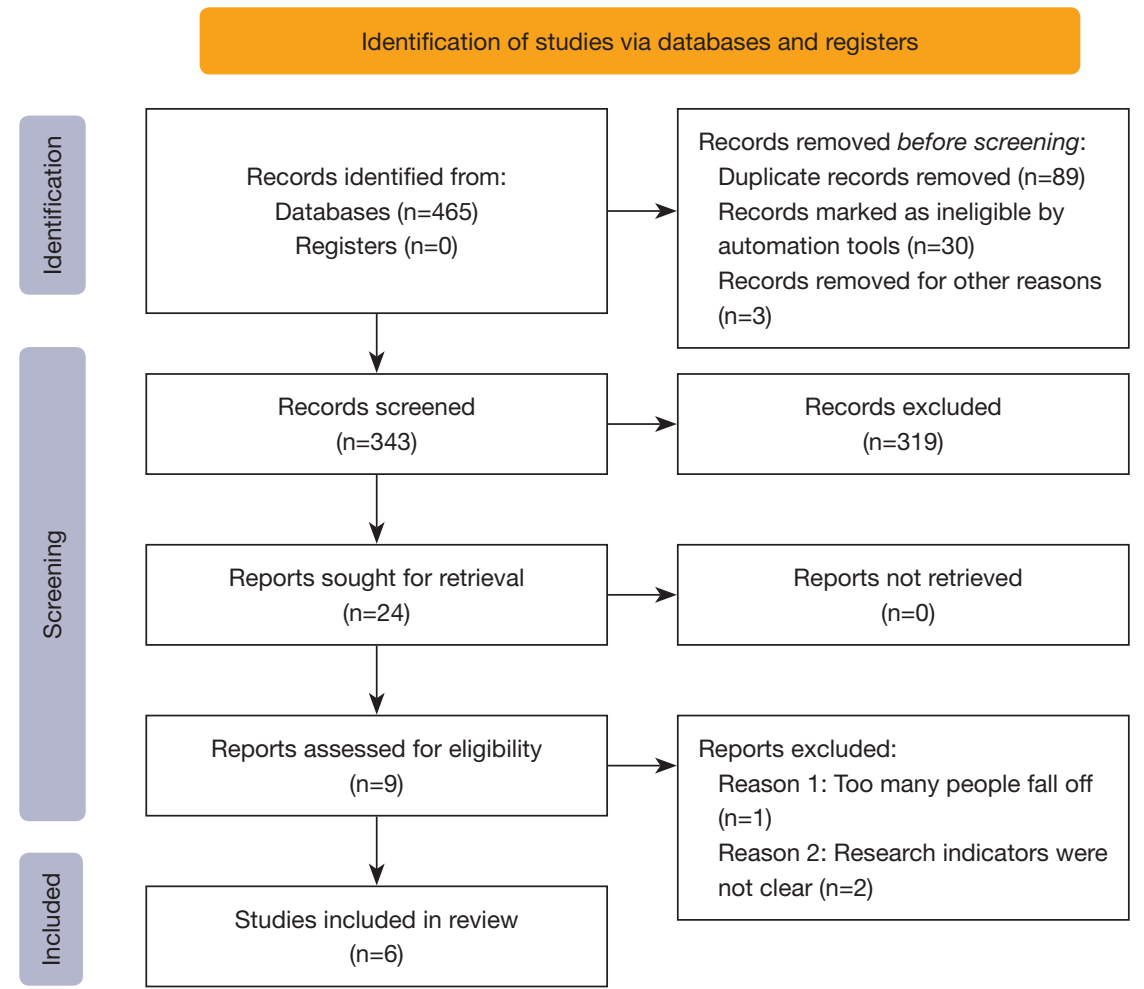

Figure 1 Flowchart of the article retrieval process.

Table 1 The basic characteristics of the included articles

\begin{tabular}{lccccc}
\hline Included articles & Year of publication & Gestational age (weeks) & Birth weight (g) & Cases (T/C) & Diagnostic criteria of BPD \\
\hline Basu et al. & 2019 & $30.9 \pm 2.9$ & $991-1,344$ & $98 / 98$ & 28 -day oxygen inhalation \\
Pearson et al. & 1992 & $27 \pm 1$ & $700-1,100$ & $27 / 22$ & 28 -day oxygen inhalation \\
Shenai et al. & 1987 & $26-30$ & $700-1,300$ & $20 / 20$ & 28 -day oxygen inhalation \\
Tyson et al. & 1999 & GA $<30$ & $700-1,300 \mathrm{~g}$ & $405 / 402$ & 36 -week oxygen inhalation \\
Wardle et al. & 2001 & $25-27$ & $<1,000 \mathrm{~g}$ & $77 / 77$ & $28-$ day or 36-week oxygen inhalation \\
Werkman et al. & 1994 & GA $<31$ & $725<$ and $<1,300$ & $15 / 15$ & 28-day oxygen inhalation \\
\hline
\end{tabular}

BPD, bronchopulmonary dysplasia.

writing manual was adopted to evaluate the risk of bias of the 6 articles included in this study. The risk of bias charts, as shown in Figures 2,3, were drawn by the RevMan 5.3 software.

\section{Incidence of 28-day BPD}

A total of 4 articles studied the incidence of 28-day BPD. Patients in the experimental group were supplemented with vitamins on the basis of routine vitamin supplementation, and subjects in the control group were given a placebo or no treatment. The results showed that $-0.17,95 \%$ CI: -0.34 to $-0.00, \mathrm{I}^{2}=76 \%$, and $\mathrm{P}=0.007$, so the REM was selected. A total of 286 infants in the vitamin A supplementation group developed BPD on the 28th day, with an incidence rate of $54 \%$, and a total of 325 infants in the control group developed BPD on the 28th day, with an incidence rate of $62 \%$. The incidence of BPD on the 28 th day was compared between the 2 groups, and it was found that the incidence of BPD in the vitamin A supplementation group 


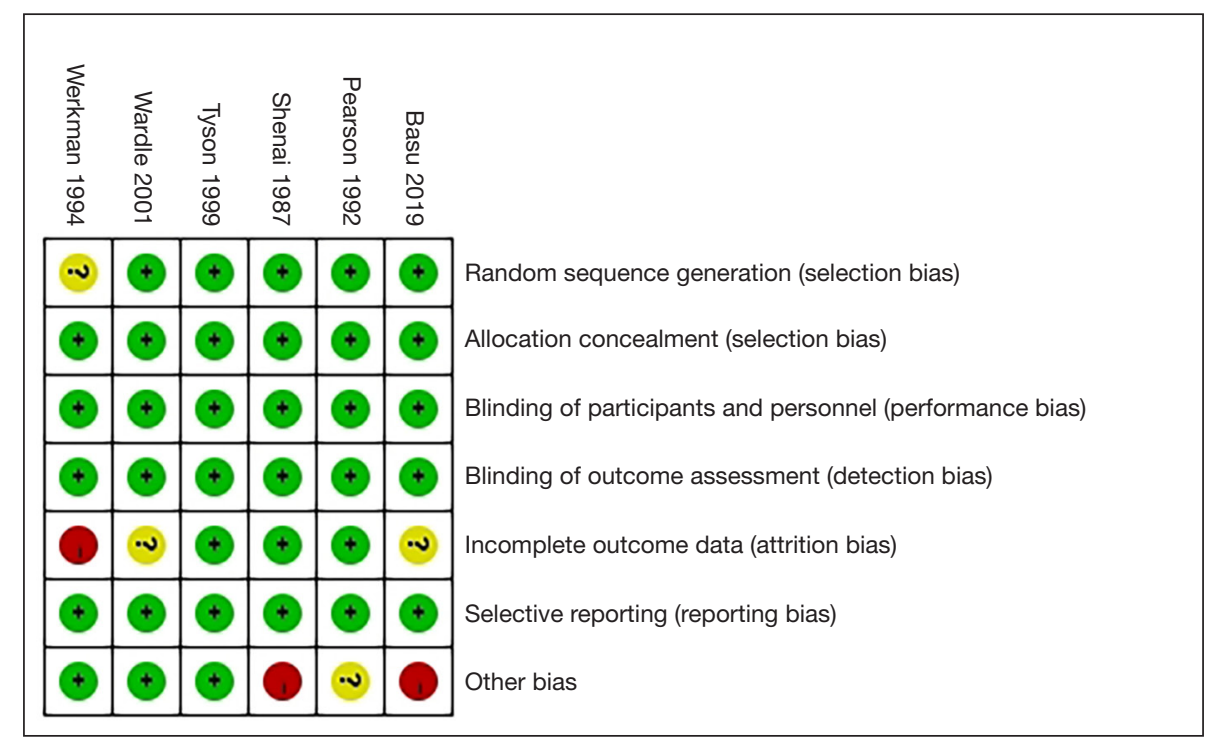

Figure 2 Assessment of the risk of bias of the included articles.

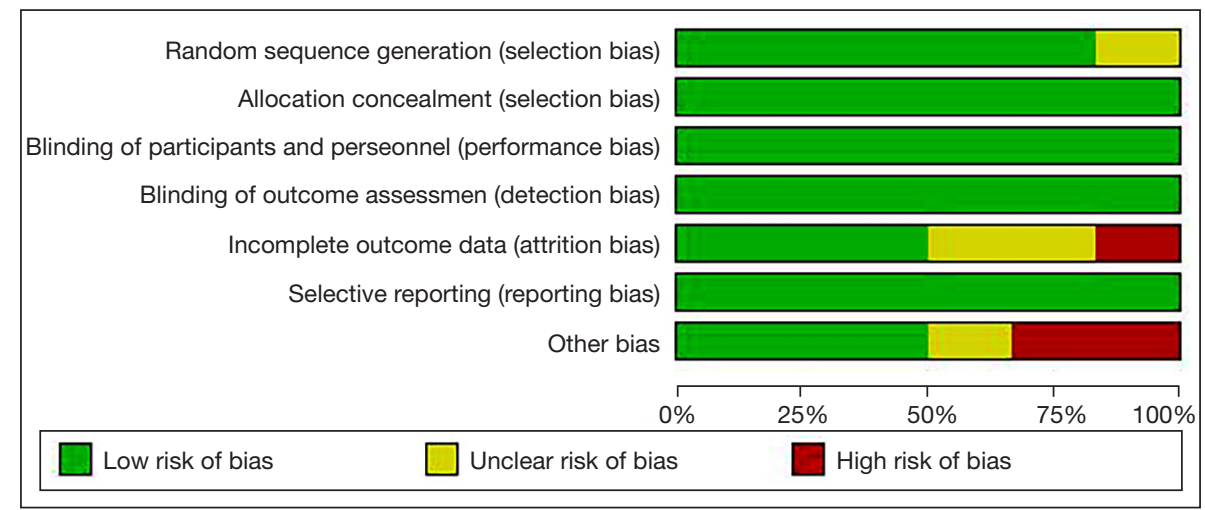

Figure 3 Multiple studies in the articles corresponded to the multiple risk bias evaluation results.

was significantly lower compared to that in the control group $(\mathrm{Z}=1.98, \mathrm{P}=0.05)$ (Figure 4). This suggests that early vitamin A supplementation has a significant impact on the occurrence of BPD in premature infants. As shown in Figure 5, the funnel plot was basically symmetrical, and most of the data was on both sides of the central axis. It shows that the included articles are reliable and there is no publication deviation.

\section{6-week survival rate}

A total of four articles studied the 36-week survival rate of infants. Patients in the experimental group were supplemented with vitamins on the basis of routine vitamin supplementation, and subjects in the control group were given a placebo or no treatment. The results showed that $1.08,95 \%$ CI: $0.80-1.46, \mathrm{P}=0.51, \mathrm{I}^{2}=0 \%$, so the FEM was selected. In the vitamin A group, 497 patients survived at 36 weeks, with A survival rate of $81.8 \%$, while in the control group, 484 patients survived, with A survival rate of $80 \%$. The 36-week survival rate was compared between the 2 groups, and it was found that the 36-week survival rate in the vitamin A supplementation group was slightly higher compared with that in the control group $(\mathrm{Z}=0.49, \mathrm{P}=0.62)$, showing no statistically significant difference (Figure 6). This suggests that early vitamin A supplementation can have a marked impact on the 36-week survival rate of premature infants. As shown in Figure 7, the funnel plot was basically 


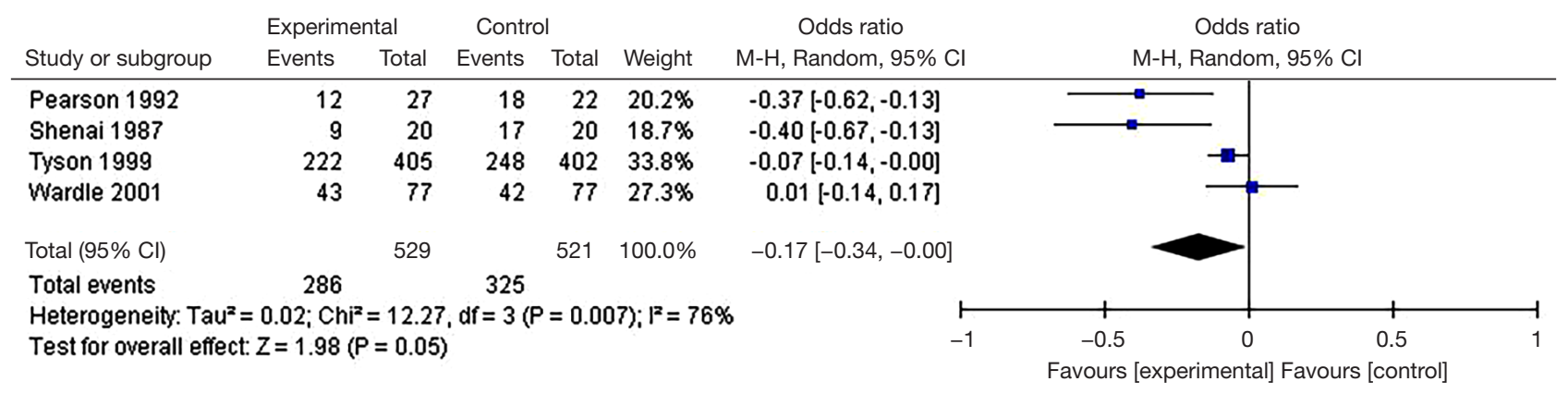

Figure 4 Forest plot of the meta-analysis on incidence of BPD on the 28th day. BPD, bronchopulmonary dysplasia.

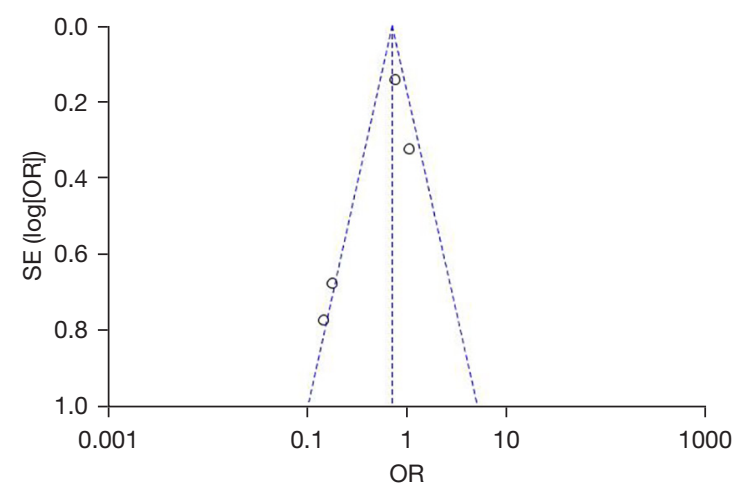

Figure 5 Funnel plot for the comparison of the incidence of BPD on the 28 th day for patients in the 2 groups. BPD, bronchopulmonary dysplasia.

\begin{tabular}{|c|c|c|c|c|c|c|c|c|}
\hline \multirow[b]{2}{*}{ Study or subgroup } & \multicolumn{2}{|c|}{ Experimental } & \multicolumn{2}{|c|}{ Control } & \multirow[b]{2}{*}{ Weight } & Odds ratio & \multirow{2}{*}{$\begin{array}{c}\text { Odds ratio } \\
\mathrm{M}-\mathrm{H} \text {, Random, } 95 \% \mathrm{Cl}\end{array}$} & \\
\hline & Events & Total & Events & Total & & $\mathrm{M}-\mathrm{H}$, Random, $95 \% \mathrm{Cl}$ & & \\
\hline Basu 2019 & 89 & 98 & 82 & 98 & $9.4 \%$ & $1.93[0.81,4.61]$ & 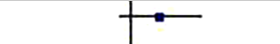 & \\
\hline Pearson 1992 & 22 & 27 & 18 & 22 & $4.6 \%$ & $0.98[0.23,4.19]$ & & \\
\hline Tyson 1999 & 346 & 405 & $34 ?$ & 402 & $63.6 \%$ & $0.93[0.63,1.38]$ & & \\
\hline Wardle 2001 & 40 & 77 & 37 & 77 & $22.3 \%$ & $1.17[0.62,2.20]$ & & \\
\hline Total $(95 \% \mathrm{Cl})$ & & 607 & & 599 & $100.0 \%$ & $1.08[0.80,1.46]$ & & \\
\hline Total events & 497 & & 484 & & & & & \\
\hline $\begin{array}{l}\text { Heterogeneity: } \mathrm{Chi}^{2} \\
\text { Test for overall effe }\end{array}$ & $\begin{array}{l}2.34, \mathrm{df}= \\
Z=0.49\end{array}$ & $\begin{array}{l}P=0 \\
0.62\end{array}$ & $51) ; I^{2}=0$ & & & 001 & $\begin{array}{ccc}0.1 & 1 & 10 \\
\text { xperimental] } & \text { Favours [cc }\end{array}$ & 1000 \\
\hline
\end{tabular}

Figure 6 Forest plot of the meta-analysis on the 36-week survival rate.

symmetrical, and most of the data was on both sides of the central axis. Such results suggest that publication bias is effective.

\section{Incidence of the 28-day oxygen uptake rate}

A total of 3 articles met the inclusion criteria. Patients in the experimental group were supplemented with vitamins on the basis of routine vitamin supplementation, and subjects in the control group were given a placebo or no treatment. The results showed that $0.81,95 \%$ CI: $0.38-1.73, \mathrm{P}=0.03$, $\mathrm{I}^{2}=70 \%$. According to the previous $\mathrm{I}^{2}=70 \%>50 \%$, the FEM was selected. Among the 530 infants in the experimental group, there were 296 cases requiring oxygen inhalation at the 28th day (accounting for $55.8 \%$ ), and 307 of 522 infants in the control group required oxygen inhalation on the 


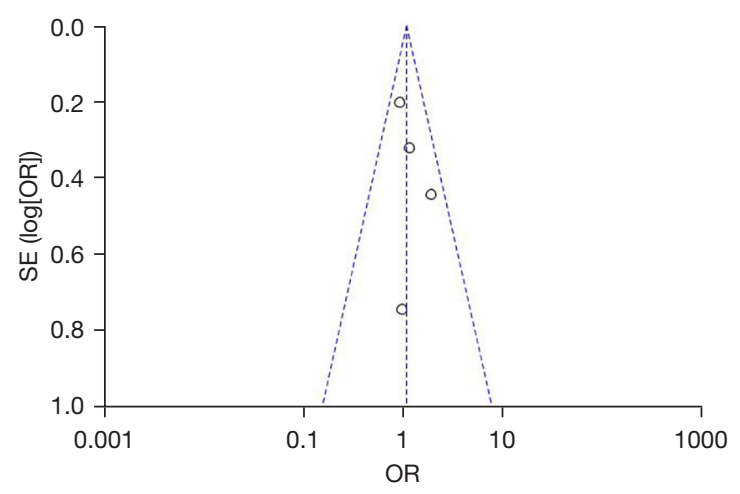

Figure 7 Funnel plot of the comparison of the 36-week survival rate between patients in the two groups.

\begin{tabular}{|c|c|c|c|c|c|c|c|c|c|}
\hline \multirow[b]{2}{*}{ Study or subgroup } & \multicolumn{2}{|c|}{ Experimental } & \multicolumn{2}{|c|}{ Control } & \multirow[b]{2}{*}{ Weight } & \multicolumn{2}{|l|}{ Odds ratio } & \multirow{2}{*}{$\begin{array}{l}\text { Odds ratio } \\
\mathrm{M}-\mathrm{H}, \text { Random, } 95 \% \mathrm{Cl}\end{array}$} & \\
\hline & Events & Total & Events & Total & & M-H, Random, 95\% Cl & & & \\
\hline Basu 2019 & 11 & 98 & 25 & 98 & $32.4 \%$ & $0.37[0.17,0.80]$ & & $\rightarrow$ & \\
\hline Pearson 1992 & 20 & 27 & 13 & 22 & $21.7 \%$ & $1.98[0.59,6.63]$ & & & \\
\hline Tyson 1999 & 265 & 405 & 269 & 402 & $45.8 \%$ & $0.94[0.70,1.25]$ & & & \\
\hline Total $(95 \% \mathrm{Cl})$ & & 530 & & 522 & $100.0 \%$ & $0.81[0.38,1.73]$ & & & \\
\hline Total events & 296 & & 307 & & & & & & \\
\hline \multicolumn{6}{|c|}{$\begin{array}{l}\text { Heterogeneity: } \operatorname{Tau}^{2}=0.30 ; \mathrm{Chi}^{2}=6.72, \mathrm{df}=2(P=0.03) ; \mathrm{I}^{2}=30 \% \\
\text { Test for overall effect: } Z=0.53(P=0.59)\end{array}$} & & 0.001 & $\begin{array}{ccc}0.1 & 1 & 10 \\
\text { [experimental] } & \text { Favours [c }\end{array}$ & 1000 \\
\hline
\end{tabular}

Figure 8 Forest plot of the meta-analysis on the incidence of the 28-day oxygen uptake rate.

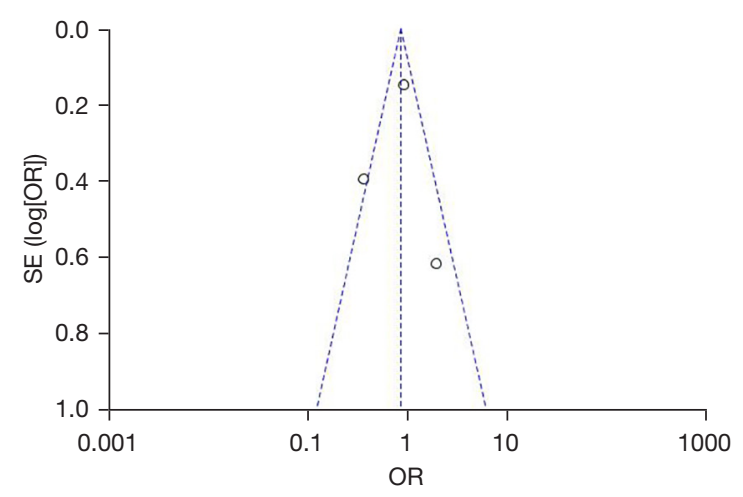

Figure 9 Funnel plot for the comparison of the incidence of the 28-day oxygen uptake rate.

28th day (accounting for 58.8\%). Therefore, the number of infants requiring oxygen inhalation on the 28th day in the experimental group was slightly lower compared with the control group $(\mathrm{Z}=0.53$ and $\mathrm{P}=0.59)$, and the difference was not statistically significant (Figure 8). As shown in Figure 9, the funnel plot was basically symmetrical, and most of the data was on both sides of the central axis. This shows that publication bias is effective.

\section{8-day ventilator use}

A total of 3 articles met the inclusion criteria. Patients in the experimental group were supplemented with vitamins on the basis of routine vitamin supplementation, and subjects in the control group were given a placebo or no treatment. The results showed that $0.77,95 \% \mathrm{CI}$ : $0.31-1.92, \mathrm{P}=0.10, \mathrm{I}^{2}=57 \%$. According to the previous 


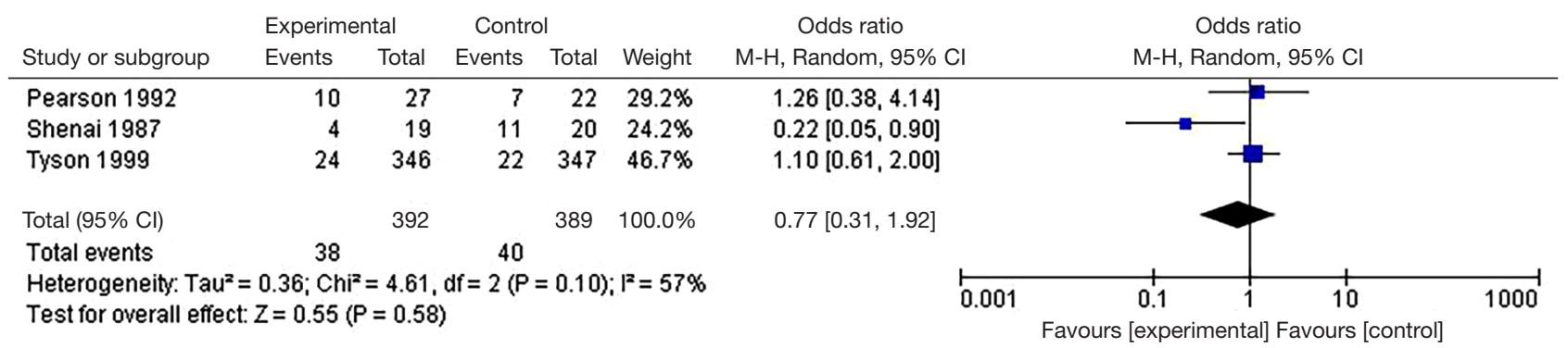

Figure 10 Forest plot of the meta-analysis on the incidence of 28-day ventilator use.

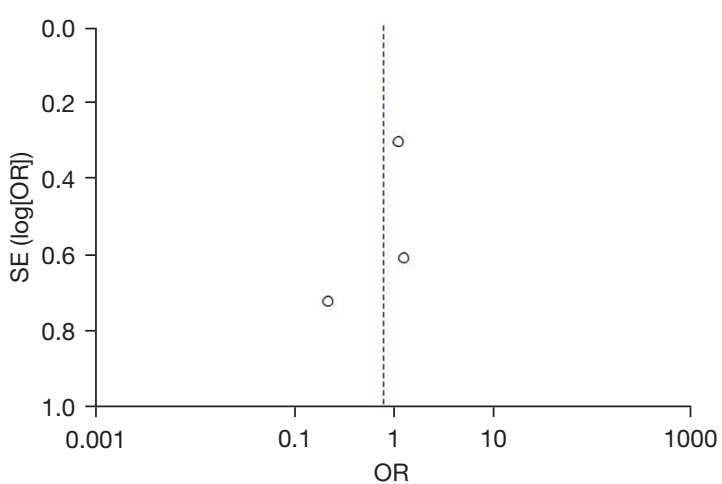

Figure 11 Funnel plot for the comparison of the incidence of 28-day ventilator use.

$\mathrm{I}^{2}=57 \%>50 \%$, the REM was selected. There were 38 cases that required ventilator use at the 28th day (accounting for $9.6 \%$ ) in the experimental group, and 40 infants in the control group required ventilator use on the 28th day (accounting for 10.2\%). Therefore, the number of infants requiring ventilator use on the 28th day in the experimental group was slightly lower compared to the control group $(\mathrm{Z}=0.55$ and $\mathrm{P}=0.58)$, and the difference was not statistically significant (Figure 10). As shown in Figure 11, the funnel plot was basically symmetrical, and most of the data was on both sides of the central axis. This shows that publication bias is effective.

\section{Days of mechanical ventilation}

A total of 3 articles met the inclusion criteria. Patients in the experimental group were supplemented with vitamins on the basis of routine vitamin supplementation, and subjects in the control group were given a placebo or no treatment. The results showed that $0.02,95 \% \mathrm{CI}:-1.46-1.49, \mathrm{P}=0.31$, $\mathrm{I}^{2}=15 \%$. According to the previous $\mathrm{I}^{2}=15 \%<50 \%$, the FEM was selected. The days of mechanical ventilation in the experimental group were slightly lower compared with the control group, showing no statistical difference $(\mathrm{Z}=0.02$ and $\mathrm{P}=0.98$ ), as illustrated in Figure 12. As shown in Figure 13, the funnel plot was basically symmetrical, and most of the data was in the range of the $95 \%$ CI. This shows that publication bias is effective.

\section{Incidence of PDA}

A total of 3 articles met the inclusion criteria. Patients in the experimental group were supplemented with vitamins on the basis of routine vitamin supplementation, and subjects in the control group were given a placebo or no treatment. The results showed that $0.77,95 \%$ CI: $0.27-2.21, \mathrm{P}=0.09$, $\mathrm{I}^{2}=67 \%$. According to the previous $\mathrm{I}^{2}=67 \%>50 \%$, the FEM was selected. Among the 190 infants in the experimental group, 36 cases suffered from PDA (accounting for 18.9\%), and 46 of 190 infants in the control group suffered from PDA (accounting for $24.2 \%$ ). Therefore, the number of infants who suffered from PDA in the experimental group was slightly lower in contrast to the control group $(\mathrm{Z}=0.482$ and $\mathrm{P}=0.63)$, and the difference was not statistically significant (Figure 14). As shown in Figure 15, the funnel plot was basically symmetrical, and most of the data was on both sides of the central axis. This shows that publication bias is effective.

\section{Discussion}

BPD is also known as CLD (18). The commonly used clinical definitions of the disease are as follows: at 36 weeks of positive gestational age: mechanical ventilation or continuous positive airway pressure or inhaled oxygen score $\geq 0.33$ is required to maintain blood oxygen saturation at $90-96 \%$; when inhaled oxygen fraction $<0.3$ maintain 


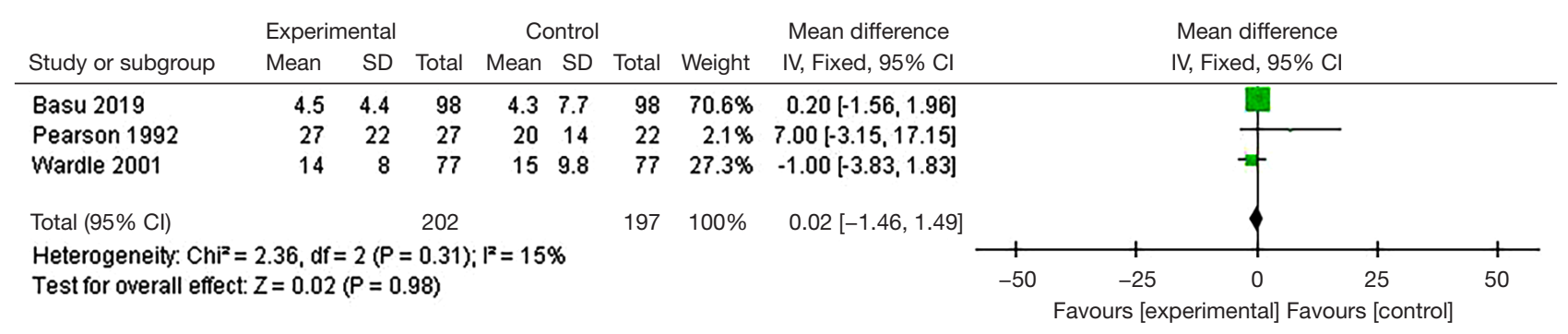

Figure 12 Forest plot of the meta-analysis on the days of mechanical ventilation.

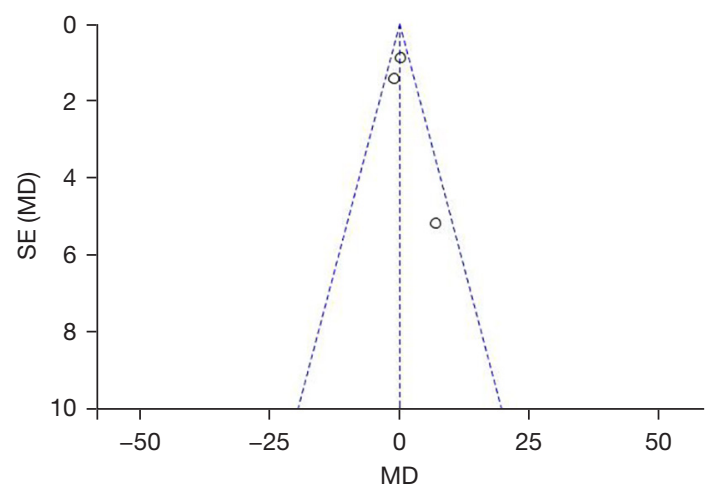

Figure 13 Funnel plot for the comparison of the days of mechanical ventilation.

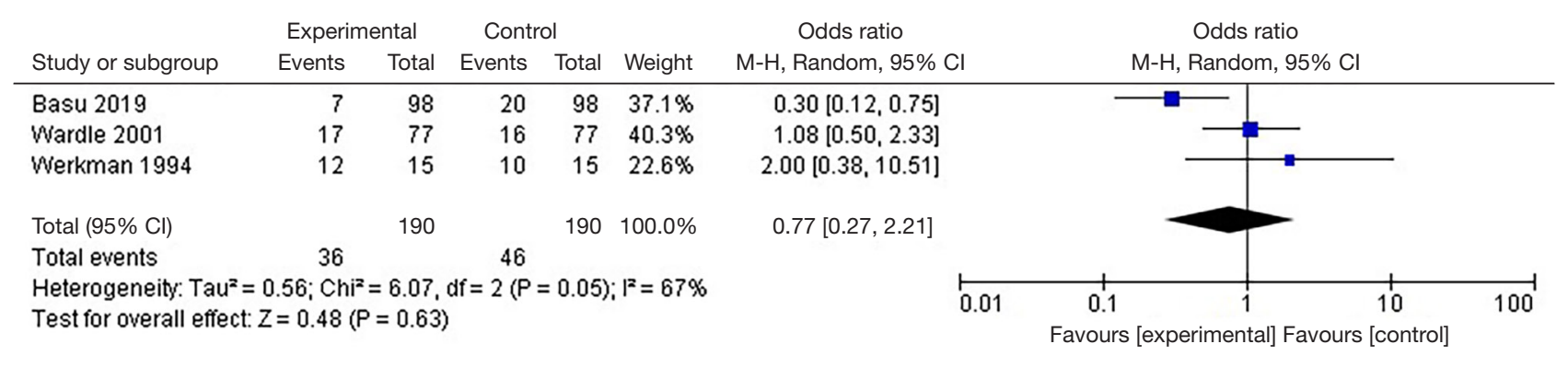

Figure 14 Forest plot of the meta-analysis on the incidence of PDA. PDA, patent ductus arteriosus.

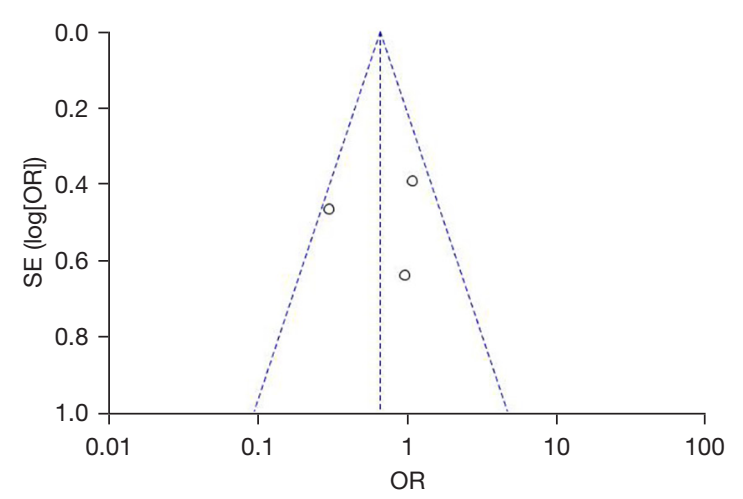

Figure 15 Funnel plot for the comparison of the incidence of PDA. PDA, patent ductus arteriosus. 
blood oxygen saturation of $90-96 \%$ or inhaled oxygen fraction $\geq 0.3$ and blood oxygen saturation $>96 \%$, it has to gradually reduce the oxygen concentration to air oxygen concentration for 30 minutes and then blood oxygen saturation $<90 \%$; and infants without oxygen are not diagnosed as "physiological BPB". This definition can better predict the outcomes of children, so it has been widely recognized and applied (19). Therefore, 36 weeks and 28 days of corrected gestational age were selected as the main outcome indicators for analysis and description in this study. In recent years, with the further improvement and advancement of perinatal technology, the birth rate and survival rate of premature infants with younger gestational age and lighter weight have further increased, and the incidence of BPD has also increased. Research data shows that the incidence of premature infants with gestational age $<32$ weeks is $1.3 \%$, the incidence of BPD in premature infants surviving $24-31$ weeks is $12.5 \%$, the incidence of BPD in infants with gestational age $24-27$ weeks is $34.7 \%$, and the incidence of BPD is $34.7 \%$. The rate gradually decreases with the increase of gestational age, and the incidence of BPD is higher and has a trend of increasing year by year $(20,21)$.

The fatality rate of BPD is relatively high, and the consequent adverse reactions last for a long time. Research data show that the effects of BPD on the respiratory system cover infancy, childhood, and adulthood. Studies have pointed out that school-age children who have suffered from BPD still have lung structural abnormalities, and severe BPD is more obvious and often complicated by pulmonary hypertension $(22,23)$. BPD is one of the difficult problems in the field of perinatal medicine, but there are still no accurate and effective treatment methods, and many treatment methods are controversial. In addition, the cost of treating BPD is relatively high, so prevention of BPD is very important. Vitamin A is an early discovered vitamin necessary to maintain the human body and metabolism. Many studies have shown that it can prevent a variety of neonatal diseases including BPD (24). However, vitamins are mainly passed from the mother to the fetus through the placenta in the third trimester, so premature infants generally lack vitamin A. BPD also occurs in premature infants, which suggests that there may be a connection between the two. At present, the clinical administration methods of vitamins mainly include oral, intravenous, and intramuscular injection. In oral administration, premature infants have immature gastrointestinal function, which affects the absorption of vitamin A. In intravenous injection, vitamin $\mathrm{A}$ is easily photolyzed and adheres to the wall of the infusion device. It is unclear whether repeated intramuscular injections bring discomfort to infants and whether repeated painful stimulation during the neonatal period will affect their neurodevelopment (25). Based on the above background, the efficacy and safety of early vitamin supplementation for the prevention and treatment of BPD in premature infants were systematically evaluated in this study. The results of the study showed that the incidence of BPD, the 28-day oxygen uptake rate, the 36-week fatality rate, PDA incidence, days of mechanical ventilation, and 28-day mechanical ventilation in the experimental group were lower than those in the control group. These results suggested that vitamin supplementation for premature infants in the early stage showed a better prevention and treatment effect on BPD and did not cause sepsis.

\section{Conclusions}

A total of 6 articles were included in this study. The efficacy and safety of early vitamin supplementation for the prevention and treatment of BPD in premature infants were systematically evaluated. The results of the study showed that the incidence of BPD, the 28-day oxygen uptake rate, the 36-week fatality rate, PDA incidence, days of mechanical ventilation, and 28-day mechanical ventilation in the experimental group were lower than those in the control group. The studies demonstrated that early supplementation of vitamin A showed a better effect on the prevention and treatment of BPD in premature infants and was safer. However, there may be some limitations due to the search of English and Chinese databases and the small number of samples included in this study. In future research, the scope of the search will be further expanded.

\section{Acknowledgments}

Funding: None.

\section{Footnote}

Reporting Checklist: The authors have completed the PRISMA reporting checklist. Available at https://dx.doi. org/10.21037/tp-21-496

Conflicts of Interest: All authors have completed the ICMJE uniform disclosure form (available at https://dx.doi. org/10.21037/tp-21-496). The authors have no conflicts of 
interest to declare.

Ethical Statement: The authors are accountable for all aspects of the work in ensuring that questions related to the accuracy or integrity of any part of the work are appropriately investigated and resolved.

Open Access Statement: This is an Open Access article distributed in accordance with the Creative Commons Attribution-NonCommercial-NoDerivs 4.0 International License (CC BY-NC-ND 4.0), which permits the noncommercial replication and distribution of the article with the strict proviso that no changes or edits are made and the original work is properly cited (including links to both the formal publication through the relevant DOI and the license). See: https://creativecommons.org/licenses/by-nc-nd/4.0/.

\section{References}

1. Rakshasbhuvankar AA, Simmer K, Patole SK, et al. Enteral Vitamin A for Reducing Severity of Bronchopulmonary Dysplasia: A Randomized Trial. Pediatrics 2021;147:e2020009985.

2. Principi N, Di Pietro GM, Esposito S. Bronchopulmonary dysplasia: clinical aspects and preventive and therapeutic strategies. J Transl Med 2018;16:36.

3. Bonadies L, Zaramella P, Porzionato A, et al. Present and Future of Bronchopulmonary Dysplasia. J Clin Med 2020;9:1539.

4. Mandell EW, Kratimenos P, Abman SH, et al. Drugs for the Prevention and Treatment of Bronchopulmonary Dysplasia. Clin Perinatol 2019;46:291-310.

5. Araki S, Kato S, Namba F, et al. Vitamin A to prevent bronchopulmonary dysplasia in extremely low birth weight infants: a systematic review and meta-analysis. PLoS One 2018;13:e207730.

6. Poets CF, Lorenz L. Prevention of bronchopulmonary dysplasia in extremely low gestational age neonates: current evidence. Arch Dis Child Fetal Neonatal Ed 2018;103:F285-91.

7. Yadav B, Sasidharan R, Gupta N. Oral vitamin A for prevention of bronchopulmonary dysplasia. Eur J Pediatr 2019;178:1601.

8. Jensen EA, Roberts RS, Schmidt B. Drugs to Prevent Bronchopulmonary Dysplasia: Effect of Baseline Risk on the Number Needed to Treat. J Pediatr 2020;222:244-7.

9. Basu S, Khanna P, Srivastava R, et al. Oral vitamin A for prevention of mortality and bronchopulmonary dysplasia.
Eur J Pediatr 2019;178:1603.

10. Gelfand CA, Sakurai R, Wang Y, et al. Inhaled vitamin A is more effective than intramuscular dosing in mitigating hyperoxia-induced lung injury in a neonatal rat model of bronchopulmonary dysplasia. Am J Physiol Lung Cell Mol Physiol 2020;319:L576-84.

11. Chandrasekaran A, Murki S. Is early oral vitamin A supplementation useful in preterm neonates at risk for bronchopulmonary dysplasia? Acta Paediatr 2020;109:634-5.

12. Basu S, Khanna P, Srivastava R, et al. Oral vitamin A supplementation in very low birth weight neonates: a randomized controlled trial. Eur J Pediatr 2019;178:1255-65.

13. Pearson E, Bose C, Snidow T, et al. Trial of vitamin A supplementation in very low birth weight infants at risk for bronchopulmonary dysplasia. J Pediatr 1992;121:420-7.

14. Shenai JP, Kennedy KA, Chytil F, et al. Clinical trial of vitamin A supplementation in infants susceptible to bronchopulmonary dysplasia. J Pediatr 1987;111:269-77.

15. Tyson JE, Wright LL, Oh W, et al. Vitamin A supplementation for extremely-low-birth-weight infants. National Institute of Child Health and Human Development Neonatal Research Network. N Engl J Med 1999;340:1962-8.

16. Wardle SP, Hughes A, Chen S, et al. Randomised controlled trial of oral vitamin A supplementation in preterm infants to prevent chronic lung disease. Arch Dis Child Fetal Neonatal Ed 2001;84:F9-F13.

17. Werkman SH, Peeples JM, Cooke RJ, et al. Effect of vitamin A supplementation of intravenous lipids on early vitamin A intake and status of premature infants. Am J Clin Nutr 1994;59:586-92.

18. Hwang JS, Rehan VK. Recent Advances in Bronchopulmonary Dysplasia: Pathophysiology, Prevention, and Treatment. Lung 2018;196:129-38.

19. Tracy MK, Berkelhamer SK. Bronchopulmonary Dysplasia and Pulmonary Outcomes of Prematurity. Pediatr Ann 2019;48:e148-53.

20. Wang SH, Tsao PN. Phenotypes of Bronchopulmonary Dysplasia. Int J Mol Sci 2020;21:6112.

21. Wu KY, Jensen EA, White AM, et al. Characterization of Disease Phenotype in Very Preterm Infants with Severe Bronchopulmonary Dysplasia. Am J Respir Crit Care Med 2020;201:1398-406.

22. Hansmann G, Sallmon H, Roehr CC, et al. Pulmonary hypertension in bronchopulmonary dysplasia. Pediatr Res 2021;89:446-55. 
23. Rysavy MA, Li L, Tyson JE, et al. Should Vitamin A Injections to Prevent Bronchopulmonary Dysplasia or Death Be Reserved for High-Risk Infants? Reanalysis of the National Institute of Child Health and Human Development Neonatal Research Network Randomized Trial. J Pediatr 2021;236:78-85.e5.

24. Dassios T, Curley A, Krokidis M, et al. Correlation of radiographic thoracic area and oxygenation impairment in

Cite this article as: Huang L, Zhu D, Pang G. The effects of early vitamin A supplementation on the prevention and treatment of bronchopulmonary dysplasia in premature infants: a systematic review and meta-analysis. Transl Pediatr 2021;10(12):3218-3229. doi: 10.21037/tp-21-496 bronchopulmonary dysplasia. Respir Physiol Neurobiol 2016;220:40-5.

25. Couroucli XI, Placencia JL, Cates LA, et al. Should we still use vitamin A to prevent bronchopulmonary dysplasia? J Perinatol 2016;36:581-5.

(English Language Editor: C. Betlazar-Maseh) 